\title{
The establishment and evaluation of rabbit model for peripherally inserted central catheter ${ }^{1}$
}

\author{
Jing ZhangI, Weiwei Yin ${ }^{\text {II }}$, Yumin Jiang ${ }^{I I I}$, Shen Juan ${ }^{\text {IV }}$ \\ DOI: http://dx.doi.org/10.1590/S0102-86502014000800003 \\ ${ }^{\mathrm{I}}$ Full Professor, Department of Nursing, Bengbu Medical College, Bengbu, China. Conception and design of the study, critical revision.

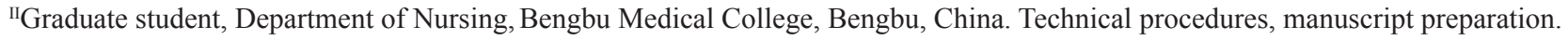 \\ IIIAssistant Professor, Department of Nursing, Bengbu Medical College, Bengbu, China. Conception and design of the study. \\ ${ }^{\mathrm{IV}}$ Graduate student, Department of Nursing, Bengbu Medical College, Bengbu, China. Technical procedures.
}

\begin{abstract}
PURPOSE: To establish a model to simulate the clinical specific process of peripherally inserted central catheter (PICC) on rabbits, and detect how long the catheter can be indwelled.

METHODS: Seventeen healthy New Zealand white rabbits were inserted the PICC according to the clinical specific procedure. With the principle of random, the rabbits were divided into four groups (14d, 21d, 28d, 35d). Each group contains four rabbits, and Group 1 was served as blank control group. When finishing the experiment, we took the blood vessels which was inserted the catheter and observed the changes of vascular endothelium using the Hematoxylin-Eosin (HE) staining.

RESULTS: $90 \%$ animals were succeeded in inserting PICC. Early signs of endovascular inflammation were predominantly neutrophils, then mainly monocytes, visible fibrous tissue hyperplasia of the vessel wall, vascular endothelial proliferation and granuloma formation. And after that the irreversible changes in the blood vessels could be observed, especially five weeks after catheterization.

CONCLUSIONS: We have succeeded in establishing the rabbit model for peripherally inserted central catheter, and provided a new way for nursing teaching and training. Since the irreversible changes of the vascular endothelium, it is recommended that the time of indwelling is not more than five weeks on animal.
\end{abstract}

Key words: Central Venous Catheters. Veins. Endothelium, Vascular. Models, Animal. Rabbits. 


\section{Introduction}

Peripherally inserted central catheter (PICC) is a deep venous catheter insertion technology. This catheter is made of silicone or polyurethane, and it can be inserted in the peripheral venous system with the catheter tip located in the superior vena cava $^{1}$. It's not only used for administration of intravenous fluid, total parenteral nutrition, anticancer agents, antibiotics, and blood products, but also for the through line blood sampling in clinical ${ }^{2}$. Compared to the traditional veinpuncture, PICC has its specific advantages such as lower risk, fewer complications, longer indwelling time and so on, and it has been accepted by health care professionals and patients.

However, insertion of thesethinnest catheters is a very delicate procedure associated with a high failure rate. During the nursing teaching and training for PICC clinical specialist, students can only learn the basic knowledge about PICC and understand the procedures, but they lack of practical training, which greatly affect the nurses to master the PICC catheterization. At the same time, researchers cannot take the patients as the research objectives directly, which interfere their in-depth mechanism research. Due to lack of reliable and high similarity of animal models, the experimental studies were restricted.

\section{Methods}

We followed the ethical code of the Council for International Organization of Medical Sciences (CIOMS) for animal experimentation.

Seventeen healthy New Zealand white rabbits (six months of age, $3 \mathrm{~kg}$ ) provided by animal experiment center of the Bengbu medical college were used in this study. All animals received a thorough physical examination before the study. Any animal that did not meet the health and weight criteria, such as any infection, inflammation, dermatitis etc, was excluded from the study. All selected animals were bred under the routine condition including ventilation, cleanliness, environmental temperature and so on.

\section{Materials}

1.9FrPICC catheter, (from Suzhou Becton Dickinson Medical Devices LTD), 20G indwelling needle, from Suzhou Becton Dickinson Medical Devices LTD), aseptic operation table, $10 \%$ chloralhydrate iodophor,75\% alcohol, tourniquet, dexamethasone, saline solution, heparin, sterile forceps, sterile scissor, alginate patches, $3 \mathrm{M}$ dressing and adhesive plaster.

The specific steps of establishment animal model for PICC

1. Rabbits were given a 12 hours of fasting prior to experiment. Anesthesia was administered with $10 \%$ chloralhydrate according to $2 \mathrm{ml} / \mathrm{kg}$ via ear vein. Anesthesia speed must be slow, and the of injection ranges from three to six minutes. At the same time, insulation measures need to be taken to prevent rabbits' temperature from dropping, which may affect the accuracy of the experiment. After anesthesia, confirmed the signs of anesthetized rabbits: eyelash and corneal reactions was abate, muscle tension was reduced, pinch skin reaction was weaken, breathing rate was between 36 and58 breathes/min, heart rate was between 140 and 200 beats/min.

2. Fixed the anesthetized rabbit on the aseptic operation table, and removed left upper limb hairs. Hairs must be clean, and then fully exposed the puncture site. Next, disinfected the site using 75\% alcohol and iodophor. The first time to disinfect using the $75 \%$ alcohol disinfection, 6 times, was to remove the skin grease and dilate blood vessels, and followed with 6 times of disinfection using the iodophor cotton balls, ensuring the maximum area of aseptic operation, and then laid the shop drapes(Figure 1A).

3. Selection of puncture site. We chose the basilica vein as the puncture site which is located in the back of the left front side of upper limb. Basilica vein is superficial, more direct and more rigid, and fewer valves. In addition, the lumen becomes thick and easy to puncture. Using a basilica vein for PICC insertion has a smaller chance of developing phlebitis than those using median cubital/cephalic vein for PICC insertion ${ }^{3}$.

4. Measured the length of the catheter in advance. Measured the length from the pre-puncture (left arm basilica vein) to the left sternoclavicular joint, and then folded down to two or three ribs. On the basis of the measuring tube length, we reserved $2 \mathrm{~cm}$ in order to reduce the waste of medical resources. We measured the length of the upper arm to detect the upper limb edema and other complications after the catheterization. Due to the rabbit's different weights, the measurements results were also different. We took the rabbit with the weight of $2.55 \mathrm{~kg}$ as a example, preset $13 \mathrm{~cm}$ as the tube length, plus $2 \mathrm{~cm}$ reserved, and the desired length is $15 \mathrm{~cm}$.

5. Preparation of operators. The PICC clinical specialist must be dressed in a hair covering, face mask, sterile gown, and sterile gloves to comply with the required maximum sterile barrier 
precautions. Flushed the talcum powder on the gloves with normal saline in order to prevent the rabbit from mechanical phlebitis.

6. Compared with the multiple lumens, the previous research showed the single lumen can decrease rates of the thrombosis and the incidence of central line associated blood stream infections $(\mathrm{CLABSI})^{4-5}$. So we chose the single lumen catheter. In order to prevent mechanical phlebitis we soaked PICC catheter for five minutes with dexamethasone $(5 \mathrm{ml}$ dexamethasone with $10 \mathrm{ml}$ normal saline solution), before veinpuncture unfold 1.9Fr PICC puncture bag ${ }^{6}$.

7. Tightened around the rabbit left upper limb using a tourniquet to make its vascular filling. Then punctured using a $20 \mathrm{G}$ indwelling needle as a peel-away sheath. Based on our experience there must be almost always a slow drip of blood from the hub of the needle when a vein is entered, and then the steel needle is withdrawn. Cutting down the connection between pintle and hose using sterile scissor, and then reserved the hose. Once the operators succeeded in placing the hose in the vein, they inserted the PICC slowly using the sterile forceps, $1 \mathrm{~cm} / \mathrm{s}$, until the placement achieved to the pre-measured depth within the vena cava. During the procedure, the speed should be slow to prevent the body from intolerance symptoms. Verified blood return, and flushed the catheter to ensure intravascular placement and patency. The catheter was secured based on the catheter manufacturer's recommendations, which is critical to preventing catheter damage (Figure 1B).

8. Cut the hose down and properly fixed, and labeled the date and time of the indwelling, catheter type, catheter length and site. Connected heparin cap joint, and then used $10 \mathrm{ml}$ heparin dilution $(100 \mathrm{u} / \mathrm{l})$ for positive pressure pulse type tube to prevent blood clotting $^{7}$.When in use, the lines were flushed with $5 \mathrm{ml}$ of normal saline before and after use(Figure 1C-E).

9. After sealing, the skin entry site was dressed with a small sterile alginate by covering $3 \mathrm{M}$ dressing to reduce the puncture point bleeding and the risk of bacterial phlebitis ${ }^{8}$. The PICC exit site dressing should be changed weekly (Figure 1F-G).

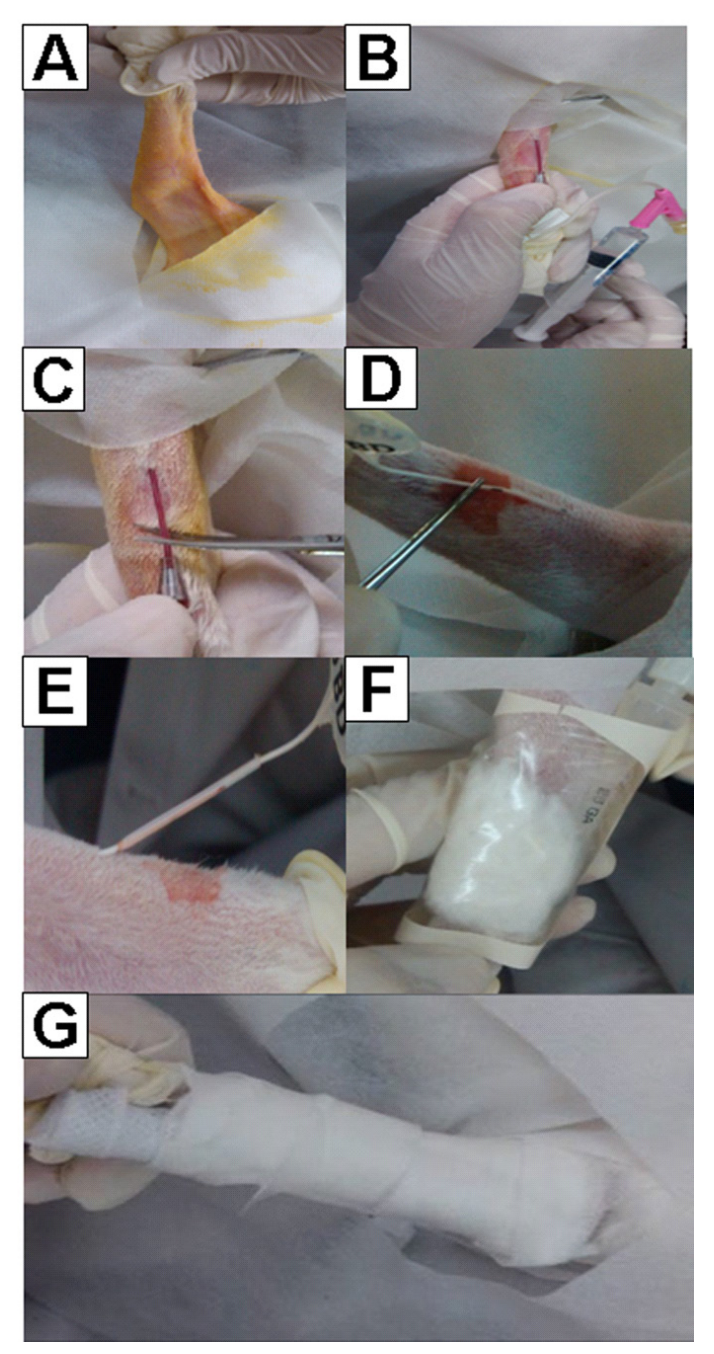

FIGURE 1 - The specific steps of establishment animal model for PICC (A-G).

\section{Evaluation of the model}

After catheterization, each rabbit was taken $\mathrm{x}$-ray to determine the position of the catheter tip. Then raised the puncture side body, and the color of the local skin should be normal. Animal survival time was just appropriate, and should meet the requirements of the follow-up tests. All animals were observed for morbidity and mortality, overt signs of toxicity (including abstinence of water), and any signs of distress throughout the study.

Animals were euthanized with an overdose of chloralhydrate at 14,21,28,35 days after catheterization. Then we used HE staining method to observe the change of vascular endothelium which was inserted catheter. 


\section{Results}

In 4 groups the total numbers of first successful punctures for the first time was 12 , and the second or more numbers for successful punctures was 4 . The total numbers of first catheterization was 13 , and the second or more numbers was 3 (Table 1). The average inserted length is $13.5 \pm 1.72 \mathrm{~cm}$, the average reserved length is $3.2 \pm 1.14 \mathrm{~cm}$, and the average operation times is $43.5 \pm 13.94 \mathrm{~min}$.

TABLE 1-The comparison of the effect of catherization (n)

\begin{tabular}{|c|c|c|c|c|}
\hline \multirow[t]{2}{*}{ Group } & \multicolumn{2}{|c|}{$\begin{array}{l}\text { number of successful } \\
\text { puncture }\end{array}$} & \multicolumn{2}{|c|}{$\begin{array}{c}\text { number of successful } \\
\text { catherization }\end{array}$} \\
\hline & first & second and more & first & second and more \\
\hline $14 d$ & 3 & 1 & 4 & 0 \\
\hline $21 d$ & 3 & 1 & 3 & 1 \\
\hline $27 d$ & 2 & 2 & 2 & 2 \\
\hline $35 d$ & 4 & 0 & 4 & 0 \\
\hline Total & 12 & 4 & 13 & 4 \\
\hline
\end{tabular}

The X-ray shows the tip of catheter was located at the rabbit superior vena cava, just as the arrow points (Figure 2).

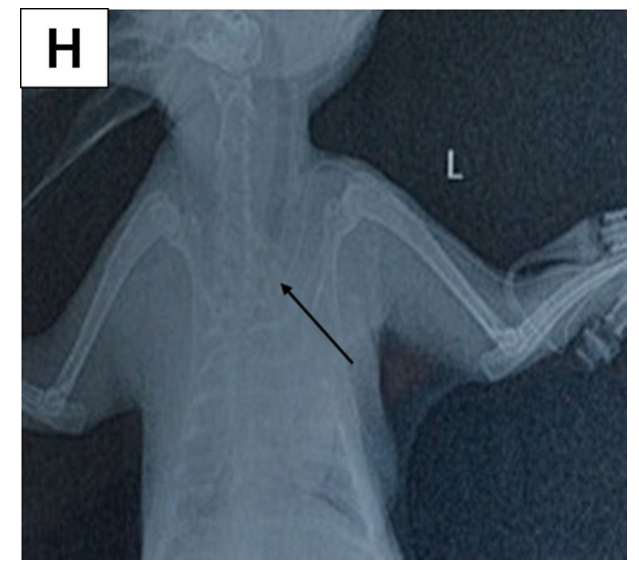

FIGURE 2 - The X-ray results (Arrow shows the location of the catheter tip).

Early endovascular inflammation were predominantly neutrophils, then mainly monocytes, visible fibrous tissue hyperplasia of the vessel wall, vascular endothelial proliferation and granuloma formation. After that, the irreversible changes in the blood vessels could be observed gradually, especially five weeks after catheterization (Figure 3).

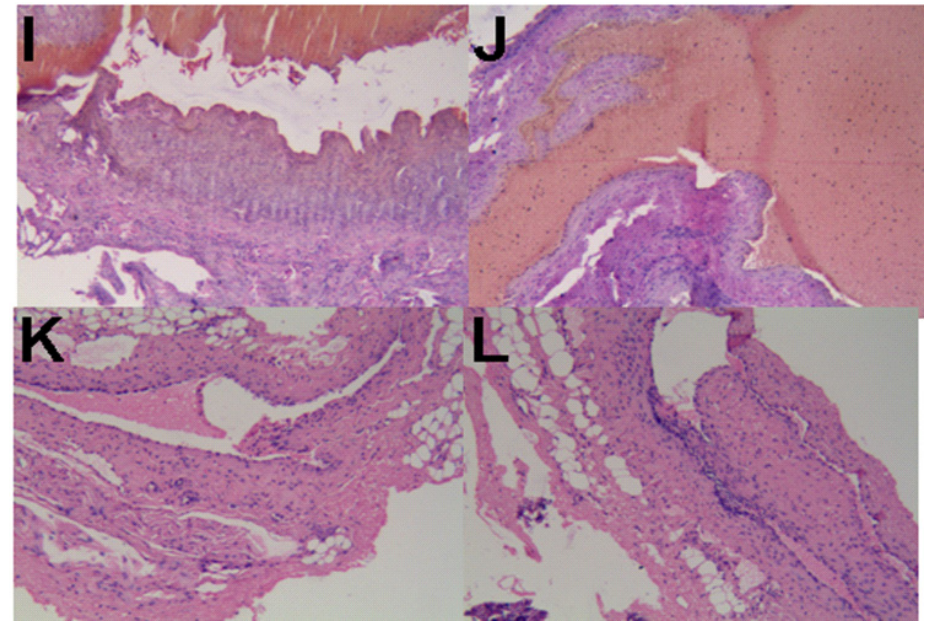

FIGURE 3 - The changes of vascular endothelial.

\section{Discussion}

The latest International Association of Nursing reported that the clinical intravenous injection should be implemented without steel needle. There is about 14 million patients using central venous infusion all over the world, and 3.3 million patients are applied with PICC for therapy, and rising rate by $10 \%$ to $20 \%$ annually ${ }^{9}$. Compared to peripheral indwelling vein (PIV), PICC has the advantages liking less reinsertion, less drug extravasation, a liquid flow rate is not influenced by position, and fewer complications related to procedure, such as lower infection rates, and decreased costs. As for the indwelling time, PIV only lien seven days, however, PICC catheter indwelling time can be up to one year ${ }^{10}$. PICC advantages over center venous catheter (CVC) include increased indwelling time, decreased venous irritation from drug therapies, particularly, reduced the physical pain of patients during their treatment, and it provides a convenient, safe and effective way for patients with venous access ${ }^{11-12}$. Besides, it greatly reduces risk of complication related to the procedure, such as pneumothorax, haemothorax and accidental arterial puncture, and it is easily performed by nurses rather than doctors, which reduce the medical costs and improves patients' health care, and could be available for outpatients ${ }^{13}$.

According to US Census Press release, the number of PICC catheter operation by nurses has researched accounted for $60 \%$ of the total, $40 \%$ of the hospitals in the United States have established the PICC groups. PICC is a difficult clinical nursing skills, so it needs specialist to perform. But in the course of PICC clinical nursing teaching and the training for the clinical specialist, students just learn the basic information of PICC through text description to understand the specific steps and nursing care after 
catheterization, and they lack of practical, hands-on training which greatly affects the nurse's master of PICC. At the same time, researchers cannot make the patients as the research objectives directly, so they are unable to carry out in-depth mechanism research. Due to lack of reliable and high similarity of animal models, the experiment studies are restricted.

The fundamental purpose of making animal model is to prevent and treat human diseases. In this study, we established the PICC animal model. It's not only to provide an practical tool for nursing teaching and training clinical specialists, but also can sort up various complications after catheterization, provide a the new clinical nursing interventions. It's the first case we choose the basilica vein as the puncture site rather than via internal and external jugular vein and back blood vessels ${ }^{14}$. At present, researchers have established the central venous catheter (CVC) models, and studied the changes of vascular endothelilum cell and inflammatory reaction protein after catheterization. Researchers from other countries have used antibiotics agents forming an anti-bacterial coating on the surface of tube to detect the occurrence of phlebitis.

On the basis of PICC animal model, we observed the complications after catheterization (such as breakage/leakage, phlebitis, catheter-related infection, thrombosis, and accidental removal. etc.) in the specific time, and the influence on the body as well. These provided theoretical foundation for PICC catheter indwelling time and the new nursing intervention measures. According to the results of the change of vascular endothelium, we found in that the early endovascular inflammation were predominantly neutrophils, then followed with monocytes, visible fibrous tissue hyperplasia of the vessel wall, vascular endothelial proliferation and granuloma formation. Finally the irreversible changes in the blood vessels occurred, especially five weeks after catheterization, so we suggest catheter indwelling time should be less than five weeks on rabbit.

There were also some restrictions in the process of establishment model. Because the rabbit blood vessels were too thin to be cleared under the ultrasound imaging, we only used the blind puncture technology, instead of the Saidingge puncture under ultrasound guidance, we need to find some ways to solve this problem.

\section{Conclusions}

The establishment of rabbit model for peripherally inserted central catheter provided a practical tool for nursing teaching and training clinical specialists, which could be widely used in the nursing field. Due to the irreversible vascular alterations five weeks after catheterization, we suggest catheter indwelling time should not be more than five weeks on rabbit.

\section{References}

1. Gibson C, Connolly BL, Moineddin R, Mahant S, Filipescu D, Amaral JG.. Peripherally inserted central venous catheters: use at tertiary care pediatric center. J Vasc Interv Radiol. 2013;24(24):132331. doi:10.1016/j.jvir.2013.04.010.

2. Chopra V, Flanders SA, Saint S. The problem with peripherally inserted central catheters. JAMA. 2012;308(15):1527-8 doi:10.1001/jama.2012.12704.

3. Shen G, Gao Y, Wang Y, Mao B, Wang X. Survey of the long-term use of peripherally inserted central venous catheters in children with cancer: experience in a developing country. J Pediatr Hematol Oncol. 2009;31(7):489-92. doi:10.1097/MPH.0b013e318190d740.

4. O'Brien J, Paquet F, Lindsay R, Valenti D.Insertion of PICCs with minimum number of lumens reduces complications and costs. J Am Coll Radiol. 2013;10(11):864-8. doi:10.1016/j.jacr.2013.06.003.

5. Evans RS, sharp JH, Linford LH, Lloyd JF, Tripp JS, Jones JP, Woller SC, Stevens SM, Elliott CG, Weaver LK. Risk of symptomatic DVT associated with peripherally inserted central catheters. Chest. 2010;138(4):803-10. doi:10.1378/chest.10-0154.

6. Weichhart T,Brandt O, Lassnig C, Müller M, Hörl WH, Stingl G, Säemann MD. The anti-inflammatory potency of dexamethasone is determined by the route of application in vivo. Immunol Lett. 2010;129(1):50-2. doi:10.1016/j.imlet.2009.12.025.

7. Sona C, Prentice D, Schallom L. National survey of central venous catheter flushing in the intensive care unit. Crit Care Nurse. 2011;32(1):e12-19. doi:10.4037/ccn2012296.

8. Chopra V, Ratz D, Kuhn L, Lopus T, Chenoweth C, Krein S. PICC-associated bloodstream infections: prevalence, patterns, and predictors. Am J Med. 2014;127(4):319-28. doi: 10.1016/j. amjmed.2014.01.001

9. Boutaric E, Gilardi M, Cécile W, Fléchelles O. Impact of clinical practice guidelines on the incidence of bloodstream infections related to peripherally inserted central venous catheter in preterm infants. Arch Pediatr. 2013;20(2):130-6. doi:10.1016/j.arcped.2012.11.001.

10. Wilson TJ, Stetler WR Jr, Fletcher JJ. Comparison of catheter-related large vein thrombosis in centrally inserted versus peripherally inserted central venous lines in the neurological intensive care unit. Clin Neurol Neurosurg. 2013;115(7):872-82. doi:10.1016/j. clineuro.2012.08.025.

11. Chopra V, O'Horo JC, Rogers MA, Maki DG, Safdar N. The risk of bloodstream infection associated with peri-pherally inserted central catheters compared with central venous catheters in adults: a systematic review and meta-analysis. Infect Control Hosp Epidemiol. 2013;34(9):908-18. doi:10.1086/671737.

12. Ibrahim Uygun, Mehmet Hanifi Okur, Selcuk Otcu, Hayrettin Ozturk. Peripherally inserted central catheters in the neonatal period. Acta Cir Bras. 2011;26(5):404-11. doi.org/10.1590/S010286502011000500014.

13. Amerasekera SS, Jones CM, Patel R, Clesby MJ. Imaging of the complications of peripherally inserted center venous catheter. Clin Radiol. 2009;64(8):832-40. doi:10.1016/j.card.2009.02.021.

14. Allan ND, Giare-Patel K, Olson ME. An in vivo rabbit model for the evaluation of antimicrobial peripherally inserted central catheter to reduce microbial migration and colonization as compared to an uncoated PICC. J Biomed Biotechnol. 2012;2012:921617. doi:10.1155/2012/921617. 
Zhang J et al.

\section{Correspondence:}

Jing Zhang

Bengbu Medical College,

Department of Nursing, Bengbu, China

Phone: +8605523175211

weiwei8906191@163.com

mini0217@126.com

Received: Mar 18, 2014

Review: May 20, 2014

Accepted: Jun 23, 2014

Conflict of interest: none

Financial source: Universities Natural Science Foundation in Anhui Province in 2014 (NO. KJ2014A165)

${ }^{1}$ Research performed at Department of Nursing, Biochemical Medicine Engineering Technology Research Center, Bengbu, China. 